\section{Adjuvant bisphosphonates in patients with breast cancer: does the potency matter?}

\author{
"Further research is warranted to determine the optimal antiresorptive \\ agent and the schedule to achieve a clinically meaningful benefit in \\ patients with early-stage breast cancer.”
}

\author{
Vito Amoroso ${ }^{*, 1}$, Fausto Petrelli², Rebecca Pedersini ${ }^{1,3}$, Edda Lucia \\ Simoncini ${ }^{3}$, Philippe Clézardin ${ }^{4}$, Sandro Barni ${ }^{2} \&$ Alfredo Berruti ${ }^{1}$
}

Breast cancer is the malignancy with the highest incidence in females, and patient clinical outcomes are steadily improving during the last decades. Early detection and advances in adjuvant therapies are the possible explanations for the observed mortality reduction.

The rationale for administering adjuvant systemic therapies in women with radically operated breast cancer is to eradicate micrometastases that are supposed to be present at the time of surgery. Disseminated tumor cells (DTCs) from breast cancer and other solid tumors can colonize the bone early during the disease course. DTCs directly compete with hematopoietic stem cells for occupancy of the osteoblastic niches in the bone marrow [1]. Since this niche allows DTCs to evade systemic anticancer therapies, the bone marrow is both a storehouse and a sanctuary for these cells that lay dormant for a considerable period of time before becoming active and able to metastasize to skeletal and extraskeletal sites [1].

Cytokines and growth factors derived from the bone microenvironment modulate the activity of DTCs in the osteoblastic niche [2].

Bisphosphonates (BPs) are analogs of pyrophosphates that can inhibit osteoclast-mediated bone resorption. As a result, BPs deprive the bone microenvironment of bone-derived growth factors that are required for seeding and growth in the bone marrow [3]. The use of these drugs in patients with early breast cancer may render the bone microenvironment less fertile for metastatic tumor growth and prolong the dormant state of DTCs within their niche.

\section{Evidence of efficacy of BPs in early-stage breast cancer}

Several randomized clinical trials have evaluated the efficacy of BPs administered in adjuvant setting in addition to chemotherapy and endocrine therapy with conflicting results [3].

Whenever the evidence is not definitive, clinical researchers increasingly rely on systematic reviews and meta-analyses to estimate the effect size of a particular treatment in a defined disease setting. Ben-Aharon et al. reported no significant difference in either disease-free survival (DFS) or overall survival (OS) in a pooled analysis of 13 randomized trials evaluating adjuvant BPs (hazard ratio [HR] of DFS: 0.95 ; 95\% CI: $0.81-1.12$; HR of OS: 0.89; 95\% CI: 0.79-1.01) [4]. This literature-based meta-analysis, however, demonstrated a positive effect on DFS for

'Medical Oncology Unit, Department of Medical \& Surgical Specialties, Radiological Sciences \& Public Health, University of Brescia at Spedali Civili Hospital, Brescia, Italy

${ }^{2}$ Medical Oncology Unit, Azienda Ospedaliera Treviglio Caravaggio, Treviglio, Italy

${ }^{3}$ Breast Unit, Spedali Civili Hospital, Brescia, Italy

${ }^{4}$ INSERM, Research Unit U1033, University of Lyon-1, Faculty of Medicine Lyon-Est (domaine Laennec), Lyon, France

*Author for correspondence: Tel.: +39030 3995410; vitoamoroso@alice.it
"Breast cancer is the malignancy with the highest incidence in females, and patient clinical
outcomes are steadily improving during the last decades."

\section{KEYWORDS \\ - bisphosphonates $\bullet$ breast cancer $\bullet$ disseminated tumor cells - osteoblastic niche $\bullet$ parathyroid hormone}


"Breast cancer is the malignancy with the highest incidence in females, and patient clinical outcomes are steadily improving during the last decades." adjuvant BPs in the subgroup of postmenopausal women [4].

More recently, the Early Breast Cancer Trials Collaborative Group (EBCTCG) presented the results of an individual patient data metaanalysis of 26 randomized trials assessing the role of adjuvant BPs in early-stage breast cancer [5]. BP therapy was associated with a modest although significant improvement in the rate of distant recurrences in a study population of more than 18,700 patients (10-year gain $1.4 \%$; $\mathrm{p}=0.03)$. However, in the postmenopausal subgroup (11,767 women), a significant $28 \%$ risk reduction of bone recurrences and a 18\% risk reduction in breast cancer mortality was reported (10-year gain in breast cancer mortality $3.3 \% ; \mathrm{p}=0.002)[5]$.

\section{Classification of BPs}

BPs are classified on the basis of whether they contain nitrogen $(\mathrm{N})$ atom or not. Non-N-BPs such as etidronate and clodronate are less potent than N-BPs (pamidronate, alendronate, risedronate, ibandronate, zoledronic acid) at inhibiting osteoclast activity [1]. The higher potency of $\mathrm{N}$-BPs, such as zoledronic acid, translates into greater efficacy in reducing the risk of skeletal complications in patients with multiple myeloma or bone metastatic breast cancer [6].

In vitro, N-BPs have shown antitumor activity, as they inhibit tumor cell adhesion, migration, invasion and proliferation and induce tumor cell apoptosis [3]. In addition, these compounds are synergistic with chemotherapeutic agents [3]. For example, in the AZURE neoadjuvant prospective randomized trial, zoledronic acid administered in combination with chemotherapy before surgery in breast cancer patients led to a higher rate of pathological complete response than chemotherapy alone [7].

N-BPs have also shown antiangiogenic effects and may have clinically relevant immunomodulatory effects through the stimulation of cytotoxic $\gamma \delta$ T-cell-mediated immune response [3].

The higher potency of N-BPs together with their direct antineoplastic, antiangiogenic and immunomodulatory activities make these drugs theoretically more efficacious than clodronate when administered in adjuvant setting.

In the EBCTCG meta-analysis, the effects of BPs on bone recurrences were similar irrespective of the type of BP employed (clodronate vs N-BPs) and the schedule (BP doses for osteoporosis vs those for cancer) [5]. These findings do not support the hypothesis of the superiority of $\mathrm{N}$-BPs over non-N-BPs in adjuvant setting.

\section{An explorative meta-analysis to assess the efficacy of different BPs}

To provide information on the effect of adjuvant $\mathrm{N}$-BPs compared with clodronate on survival in early breast cancer, we performed a literaturebased meta-analysis of 12 randomized prospective trials comparing different BPs with either placebo or no antiresorptive therapy [8-19]. For all of these studies, data on OS were reported in the most updated publication. The meta-analysis demonstrated a weak effect of adjuvant BPs on OS considering the complete set of 12 trials (HR of death: 0.92 ; $95 \%$ CI: $0.80-1.04)$ with moderate heterogeneity among trials $\left(\mathrm{I}^{2}=43 \%\right)$; this calculated effect estimate is close to the event rate ratio (BPs vs control) of breast cancer mortality reported by the EBCTG meta-analysis (rate ratio: 0.90; SE: 0.045) [5].

In four of these 12 trials (4981 patients) clodronate was employed in the experimental arm [8-11]. In eight trials (10,927 patients) an $\mathrm{N}$-BP was tested (zoledronic acid, pamidronate, ibandronate or risedronate) [12-19]. In the clodronate trial subset, patients treated with antiresorptive therapy had a nonsignificant reduction in the risk of death compared with those in the control arms (HR of death: 0.86; 95\% CI: 0.67-1.11), with a strong heterogeneity $\left(\mathrm{I}^{2}=61 \%\right)$ driven by a single trial [8]. In the N-BP trial subset, OS was not different among patients randomized to the N-BP group compared with the control group (HR of death: 0.96; 95\% CI: 0.83-1.11), with modest heterogeneity among trials $\left(\mathrm{I}^{2}=25 \%\right)$. In this analysis, the treatment effect estimate was not significantly different between the two trial subsets, suggesting that adjuvant treatment with high-potency N-BPs is not more effective on survival than adjuvant clodronate in patients with breast cancer. Interestingly, the point estimate of the calculated hazard ratio was better in the clodronate trial subset than in the N-BP trial subset ( 0.86 vs 0.96$)$.

\section{Why is the effect of adjuvant clodronate not inferior when compared with more potent N-BPs?}

The efficacy of clodronate in adjuvant setting is confirmed by the results of a recently published pharmaco-epidemiological study on 21,664 women with breast cancer [20]. This study has shown a lower risk of skeletal metastasis 
and all-cause mortality in patients receiving low-dose oral bisphosphonates for prevention or treatment of postmenopausal osteoporosis compared with those never exposed to BPs [20].

Clodronate is a weak BP that is also poorly absorbed when administered orally [10]. Therefore, the efficacy of this drug in adjuvant setting suggests that a low reduction of bone turnover could be associated with a survival impact.

It is not clear why a greater inhibition of bone turnover induced by more potent N-BPs seems to fail to translate into better outcome benefits in women with early breast cancer. One possible explanation is that the greater bone turnover inhibition induced by N-BPs can induce calcium entrapment in bone and secondary hyperparathyroidism in response to the increased calcium demand. Hyperparathyroidism after BP treatment is also favored by an hypovitaminosis $\mathrm{D}$ status that is highly prevalent in breast cancer patients that receive adjuvant therapies [21].

Parathyroid hormone (PTH) induces the expression of RANKL on osteoblasts and stromal cells in the bone marrow. RANKL binds the RANK receptor on osteoclast precursors and promotes osteoclastogenesis. In turn, bone resorption by osteoclasts releases other peptides which can promote tumor growth (i.e., TGF- $\beta$, IGFs and PDGF) [22]. PTH can also stimulate the osteoblast niche within the bone marrow. In fact, it increases the expression level of $\mathrm{N}$-cadherin on osteoblasts [23]. N-cadherin-mediated adhesion may link to the canonical Wnt and Notch1 pathway through $\beta$-catenin, and these signaling pathways are essential for hematopoietic stem cell renewal [24]. As a matter of fact, PTH stimulation can induce mobilization of hematopoietic stem cells within the osteoblast niche [24], and with the same mechanism it could also stimulate the regenerative capacity of DTCs in the niche. PTH is similar to PTH-related peptide (PTHrp) and both PTHrp and PTH share the same receptor (PTHR1) which is expressed on different types of cancers. The interaction of PTH/PTHrp with PTHR1 can promote cell proliferation and growth, resistance to apoptosis, invasion and metastasis [22]. Of note, a post hoc analysis of a randomized placebo-controlled trial evaluating zoledronic acid for metastatic prostate cancer reported that elevated serum PTH levels could predict worse outcomes in patients treated with zoledronic acid [25].

Clodronate has shown fewer direct antitumor effects than N-BPs in preclinical models [26]. Nonetheless, the weaker inhibition of osteoclastmediated bone resorption induced by clodronate may prevent the raising of serum PTH levels. Of course, this is a speculative interpretation that should be considered as a suggestion for future studies.

Overall, this explorative analysis underlines how difficult targeting the bone microenvironment to control the activity of DTCs within the osteoblastic niche is. Further research is warranted to determine the optimal antiresorptive agent and the schedule to achieve a clinically meaningful benefit in patients with early-stage breast cancer.

\section{Financial \& competing interests disclosure}

The authors have no relevant affiliations or financial involvement with any organization or entity with a financial interest in or financial conflict with the subject matter or materials discussed in the manuscript. This includes employment, consultancies, honoraria, stock ownership or options, expert testimony, grants or patents received or pending, or royalties.

No writing assistance was utilized in the production of this manuscript.

\section{References}

1 Clézardin P. Mechanisms of action of bisphosphonates in oncology: a scientific concept evolving from antiresorptive to anticancer activities. Bone Key Rep. 2, 267 (2013).

2 Weilbaecher KN, Guise TA, Mccauley LK. Cancer to bone: a fatal attraction. Nat. Rev. Cancer 11(6), 411-425 (2011).

3 Gnant M, Clézardin P. Direct and indirect anticancer activity of bisphosphonates: a brief review of published literature. Cancer Treat. Rev. 38(5), 407-415 (2012).
4 Ben-Aharon I, Vidal L, Rizel S et al. Bisphosphonates in the adjuvant setting of breast cancer therapy - effect on survival: a systematic review and meta-analysis. PLoS ONE 8(8), e70044 (2013).

5 Early Breast Cancer Trialists' Collaborative G. Adjuvant bisphosphonate treatment in early breast cancer: meta-analyses of individual patient data from randomised trials. Lancet doi:10.1016/S0140-6736(15)60908-4 (2015) (Epub ahead of print).

6 Rosen LS, Gordon D, Kaminski M et al. Long-term efficacy and safety of zoledronic acid compared with pamidronate disodium in the treatment of skeletal complications in patients with advanced multiple myeloma or breast carcinoma: a randomized, doubleblind, multicenter, comparative trial. Cancer 98(8), 1735-1744 (2003).

7 Coleman RE, Winter MC, Cameron D et al. The effects of adding zoledronic acid to neoadjuvant chemotherapy on tumour response: Exploratory evidence for direct anti-tumour activity in breast cancer. $\mathrm{Br}$. J. Cancer 102(7), 1099-1105 (2010).

8 Saarto T, Vehmanen L, Virkkunen P, Blomqvist C. Ten-year follow-up of a randomized controlled trial of adjuvant 
clodronate treatment in node-positive breast cancer patients. Acta Oncol. 43(7), 650-656 (2004).

9 Powles T, Paterson A, Mccloskey E et al. Reduction in bone relapse and improved survival with oral clodronate for adjuvant treatment of operable breast cancer [ISRCTN83688026]. Breast Cancer Res. 8(2), R13 (2006).

10 Diel IJ, Jaschke A, Solomayer EF et al. Adjuvant oral clodronate improves the overall survival of primary breast cancer patients with micrometastases to the bone marrow: a long-term follow-up. Ann. Oncol. 19(12), 2007-2011 (2008).

11 Paterson AH, Anderson SJ, Lembersky BC et al. Oral clodronate for adjuvant treatment of operable breast cancer (National Surgical Adjuvant Breast and Bowel Project Protocol B-34): a multicentre, placebo-controlled, randomised trial. Lancet Oncol. 13(7), 734-742 (2012).

12 Delmas PD, Balena R, Confravreux E, Hardouin C, Hardy P, Bremond A. Bisphosphonate risedronate prevents bone loss in women with artificial menopause due to chemotherapy of breast cancer: a double-blind, placebo-controlled study. J. Clin. Oncol. 15(3), 955-962 (1997).

13 Kristensen B, Ejlertsen B, Mouridsen HT et al. Bisphosphonate treatment in primary breast cancer: results from a randomised comparison of oral pamidronate versus no pamidronate in patients with primary breast cancer. Acta Oncol. 47(4), 740-746 (2008).
14 Leal T, Tevaarwerk A, Love R et al. Randomized trial of adjuvant zoledronic acid in postmenopausal women with high-risk breast cancer. Clin. Breast Cancer 10(6), 471-476 (2010).

15 Gnant M, Mlineritsch B, Stoeger $\mathrm{H}$ et al. Zoledronic acid combined with adjuvant endocrine therapy of tamoxifen versus anastrozol plus ovarian function suppression in premenopausal early breast cancer: final analysis of the Austrian Breast and Colorectal Cancer Study Group Trial 12. Ann. Oncol. 26(2), 313-320 (2015).

16 Brufsky AM, Harker WG, Beck JT et al. Final 5 year results of Z-FAST trial: adjuvant zoledronic acid maintains bone mass in postmenopausal breast cancer patients receiving letrozole. Cancer 118(5), 1192-1201 (2012).

17 Von Minckwitz G, Mobus V, Schneeweiss A et al. German adjuvant intergroup nodepositive study: a Phase III trial to compare oral ibandronate versus observation in patients with high-risk early breast cancer. J. Clin. Oncol. 31(28), 3531-3539 (2013).

18 Coleman R, De Boer R, Eidtmann $\mathrm{H}$ et al. Zoledronic acid (zoledronate) for postmenopausal women with early breast cancer receiving adjuvant letrozole (ZO-FAST study): final 60-month results. Ann. Oncol. 24(2), 398-405 (2013).

19 Coleman R, Cameron D, Dodwell D et al. Adjuvant zoledronic acid in patients with early breast cancer: final efficacy analysis of the AZURE (BIG 01/04) randomised open-label Phase 3 trial. Lancet Oncol. 15(9), 997-1006 (2014).

20 Kremer R, Gagnon B, Meguerditchian AN, Nadeau L, Mayo N. Effect of oral bisphosphonates for osteoporosis on development of skeletal metastases in women with breast cancer: results from a pharmacoepidemiological study. J. Natl Cancer Inst. 106(11), (2014).

21 Friedman CF, Demichele A, Su HI et al. Vitamin $\mathrm{D}$ deficiency in postmenopausal breast cancer survivors. J. Womens Health (Larchmt) 21(4), 456-462 (2012).

22 Soki FN, Park SI, Mccauley LK. The multifaceted actions of PTHrp in skeletal metastasis. Future Oncol. 8(7), 803-817 (2012).

23 Marie PJ. Role of $\mathrm{N}$-cadherin in bone formation. J. Cell Physiol. 190(3), 297-305 (2002).

24 Huber BC, Grabmaier U, Brunner S. Impact of parathyroid hormone on bone marrowderived stem cell mobilization and migration. World J. Stem Cells 6(5), 637-643 (2014).

25 Berruti A, Cook R, Saad F et al. Prognostic role of serum parathyroid hormone levels in advanced prostate cancer patients undergoing zoledronic acid administration. Oncologist 17(5), 645-652 (2012).

26 Winter MC, Holen I, Coleman RE. Exploring the anti-tumour activity of bisphosphonates in early breast cancer. Cancer Treat. Rev. 34(5), 453-475 (2008). 